\title{
Hybrid Reasoning and Coordination Methods on Multi-Agent Systems
}

\author{
S. Heras, M. Navarro and V. Julián
}

\begin{abstract}
This paper briefly introduces a summary of the special session on Hybrid Reasoning and Coordination Methods on Multi-Agent Systems, held in conjunction with the 4th International Conference on Hybrid Artificial Intelligence Systems 2009 (HAIS'09). The research papers of this session have been revised and extended and the final results are published in this journal special issue.
\end{abstract}

Index Terms-Hybrid Multi-Agent Systems, Reasoning and Coordination Methods

\section{INTRODUCTION}

Over the last years, Multi-Agent Systems (MAS) have been successfully applied to manage complex distributed processes in a wide range of application domains. In these systems, agents must be able to reason autonomously and coordinate their activities with other agents of the system to fulfil their objectives. Since the consolidation of the MAS paradigm in the 90's, much research has been done to provide social agents with better reasoning and coordination mechanisms that enhance their intelligence and improve their performance. Recently, the research community in MAS has focused their efforts on adapting MAS to open environments where heterogeneous agents interact. In these systems, agents can enter in (or leave) the system, form societies and communicate with other agents. Common assumptions about the agents of most MAS, such as honesty, cooperativeness and trustworthiness cannot be longer taken as valid hypothesis in open MAS. Therefore, the high dynamism of these open MAS gives rise to a greater need for complex reasoning and coordination mechanisms to control the access to the system and the deliberative processes of the agents.

The methods applied are very varied and the synergies with different areas of the Artificial Intelligence and other sciences have given rise to excellent results. Hybrid Artificial Intelligence Systems (HAIS) combine symbolic and sub-symbolic techniques to provide hybrid problem solving models. Their capabilities in handling many real world complex problems, involving imprecision, uncertainty and high-dimensionality makes them very suitable to cope with reasoning and coordination problems in complex open MAS.

The special session on Hybrid Multi-Agent Systems, Reasoning and Coordination Methods was aimed at discussing research on HAIS to develop reasoning and coordination methods on MAS. The session was conceived as a forum to

Stella Heras, Martí Navarro and Vicente Julián are with Universidad Politécnica de Valencia. S. Heras: sheras@dsic.upv.es

M. Navarro: mnavarro@dsic.upv.es

V. Julián: vinglada@dsic.upv.es present theoretical advances and real-world applications in this multidisciplinary research field.

\section{Special Issue on Hybrid Multi-Agent Systems, REASONING AND COORDINATION METHODS}

This volume presents a revised version of the best papers presented in the special session on Hybrid Multi-Agent Systems, Reasoning and Coordination Methods, held in conjunction with the 4th International Conference on Hybrid Artificial Intelligence Systems 2009 (HAIS'09). Section encouraged topics, such as: practical reasoning methods, multi-agent casebased reasoning, artificial social systems, trust and reputation, social and organizational structure, teamwork, coalition formation, distributed problem solving, electronic markets and institutions, cooperative and non-cooperative game theory methods, social choice theory, voting protocols, auction and mechanism design, argumentation, negotiation and bargaining, agent commitments, semantic alignment and ontologies were tackled by the session papers, advancing research on the area of hybrid artificial intelligence systems. Each paper was reviewed by two independent experts in this area.

In the first paper, Muñoz and Botía propose an Argumentation System Based on Ontologies (ASBO) to cope with conflicts based on inconsistent knowledge which arise when agents exchange information. Their formal model follows an engineering-oriented approach to develop a software architecture which allows working with argumentation in MAS.

In the second paper, Pinzón et al. presents the core component of a solution based on agent technology that allows the identification of denial of service (DoS) attacks introduced in the XML of SOAP messages. The paper presents an advanced classification mechanism designed in two phases that are incorporated within a CBR-BDI Agent type. In addition, the method involves the use of decision trees, fuzzy logic rules and neural networks for filtering attacks. As a result of this work, a prototype was developed and the conclusions obtained are presented in the paper.

In the third paper, Búrdalo et al. propose a general tracing system that could be used by agents in the system to trace other agents' activity. This provides agents with an alternative way for perceiving their environment. The paper presents preliminary results of the authors' work, consisting of the requirements which should be taken into account when designing such a tracing system.

In the fourth paper, Castillo et al. show their work on developing a Multi-Agent Recommendation System (RecMAS) able to coordinate the interactions between a user agent and a set of commercial agents. The system provides a useful service 
for monitoring changes in the user agents beliefs and decisions based on two parameters: (i) the strength of its own beliefs and (ii) the strength of the commercial agents suggestions. The system was used to test a prototype that copes with several commercial activities in a real shopping centre by using wireless devices (PDA, mobile phone, etc.). Using a theoretical model and the simulation experiments, commercial strategies in relation with the socio-dynamics of the system were obtained.

In the fifth paper, Heras et al. propose a new dialogue game protocol for modelling the interactions produced between the agents of an open MAS that must reach an agreement on the use of norms. The protocol is formally specified and the decision-making process of the agents is also developed. In addition, the authors provide an application example for showing both the performance of the protocol and its usefulness as a mechanism for managing the solving process of a coordination problem through norms.

Finally, in the last paper Navarro et al. copes with the problem of merging intelligent deliberative techniques with real-time reactive actions in the special context of RealTime Multi-Agent Systems (RTMAS). In these systems, the temporal restrictions of their Real-Time Agents make their deliberation process to be temporally bounded. The paper proposes a solution based on a temporally bounded CaseBased Reasoning mechanism. Thus, it also presents a guide to adapt the Case-Based Reasoning cycle to be used as deliberative mechanism for Real-Time Agents.

\section{SPECIAL ISSUE ACKNOWLEDGEMENTS}

We would like to thank authors for their valuable work and their interest in the special session on Hybrid MultiAgent Systems, Reasoning and Coordination Methods. Their contributions show high quality research in the area and their presentations gave rise to interesting discussions.

This special session could not be held without the support of the organising committee of HAIS 2009, to whom we are very grateful for their invaluable help. We also want to thank the experts of the program committee for their independent reviews and comments to the authors.

Finally, the Guest Editors wish to specially thank Professors Miguel Cazorla and Vicente Matellan (Editors-in-Chief of the Journal of Physical Agents) for the publication of this special issue and their support during the publishing process.

\section{ACKNOWLEDGEMENTS}

This special session was supported by CONSOLIDERINGENIO 2010 under grant CSD2007-00022 and by the Spanish government and GVA funds under TIN2006-14630C0301 and PROMETEO/2008/051 projects. 